\title{
Muons as Local Probes of Three-Body Correlations in the Mixed State of Type-II Superconductors
}

\author{
G. I. Menon, ${ }^{1}$ A. Drew, ${ }^{2, *}$ U. K. Divakar, ${ }^{2}$ S. L. Lee, ${ }^{2}$ R. Gilardi, ${ }^{3}$ J. Mesot, ${ }^{3}$ F. Y. Ogrin,${ }^{4}$ D. Charalambous, ${ }^{5, \dagger}$ \\ E. M. Forgan, ${ }^{5}$ N. Momono, ${ }^{6}$ M. Oda,${ }^{6}$ C. Dewhurst, ${ }^{7}$ and C. Baines ${ }^{8}$ \\ ${ }^{1}$ The Institute of Mathematical Sciences, CIT Campus, Taramani, Chennai 600 113, India \\ ${ }^{2}$ School of Physics and Astronomy, University of St. Andrews, Fife KY16 9SS, United Kingdom \\ ${ }^{3}$ Laboratory for Neutron Scattering, ETH Zurich and PSI Villigen, CH-5232 Villigen PSI, Switzerland \\ ${ }^{4}$ Department of Physics, University of Exeter, Exeter EX4 4QL, United Kingdom \\ ${ }^{5}$ School of Physics and Astronomy, University of Birmingham, Birmingham B15 2TT, United Kingdom \\ ${ }^{6}$ Department of Physics, Hokkaido University, Sapporo 060-0810, Japan \\ ${ }^{7}$ Institut Laue-Langevin, 6 rue Jules Horowitz, BP 156-38042, Grenoble Cedex, France \\ ${ }^{8}$ Laboratory for Muon Spin Spectroscopy, PSI Villigen, CH-5232 Villigen PSI, Switzerland
}

(Received 30 March 2005; revised manuscript received 18 April 2006; published 27 October 2006)

The vortex glass state formed by magnetic flux lines in a type-II superconductor is shown to possess nontrivial three-body correlations. While such correlations are usually difficult to measure in glassy systems, the magnetic fields associated with the flux vortices allow us to probe these via muon-spin rotation measurements of the local field distribution. We show via numerical simulations and analytic calculations that these observations provide detailed microscopic insight into the local order of the vortex glass and more generally validate a theoretical framework for correlations in glassy systems.

DOI: 10.1103/PhysRevLett.97.177004

In systems which possess long-range order, such as atomic crystals, the local arrangement of particles is easily obtained from scattering experiments. In disordered systems, the average correlation between the positions of two particles can be measured by scattering techniques, but inferring anything more about the local geometry is a far more subtle issue. Little is known experimentally about correlation functions of higher order. Measurements of three-body correlation functions for colloids imaged in a quasi-two-dimensional geometry have been reported recently [1]. However, bulk measurements of three-body correlation functions in any system are still unavailable and our understanding of such correlations is derived mainly from simulations. This Letter reports a study of the local structure of the vortex glass phase in a bulk type-II superconductor. The vortex glass phase provides an example of a glassy system where the local geometry in the bulk is uniquely amenable to investigation, due to the magnetic fields associated with the vortices, which we measure by the muon-spin rotation ( $\mu \mathrm{SR})$ technique. By coupling these measurements with Monte Carlo simulations and analytic calculations, we demonstrate both the existence of nontrivial three-body correlations in the fluxline array and a theoretical framework in which they may be understood.

In the mixed state of a type-II superconductor, an applied magnetic field penetrates as lines of magnetic flux, quantized in units of the flux quantum $\Phi_{0}=h / 2 e$. Such vortex lines would form an Abrikosov flux lattice at low temperatures in the absence of quenched disorder. As the temperature or the strength of disorder is increased, ordered arrangements of vortex lines yield to disordered ones $[2,3]$. Weak quenched disorder converts the crystal into a
PACS numbers: 74.25.Qt, 61.20.Gy, 74.72.Dn, 76.75.+i

"Bragg glass" with quasi-long-range order in translational correlations [4]. At stronger disorder, "vortex glass" states with short-ranged correlations are obtained. Neutron scattering measurements support the proposal of a power-law decay of translational correlations in the Bragg glass phase [5]. In contrast, structure and correlations in vortex glasses remain little understood.

Our experimental system is $\mathrm{La}_{1.9} \mathrm{Sr}_{0.1} \mathrm{CuO}_{4-\delta}$ (LSCO), an underdoped high- $T_{c}$ superconductor with properties which amplify the effects of thermal fluctuations and quenched disorder. It was recently shown using $\mu \mathrm{SR}$ measurements, on the same high quality crystal as used in this experiment, that there is a field induced transition in LSCO to a vortex glass phase [6]. Our present $\mu$ SR experiments were performed using the GPS spectrometer at PSI, Switzerland with the field nearly parallel to the $c$ axis of the crystal. The experimental arrangement was as described in Ref. [6]. The novelty of the present work is that by relating the third moment of the magnetic field distribution to an integral over a three-particle structure factor, we are able to provide information about three-body correlations in both the ordered and the glassy phases.

In a $\mu$ SR experiment, the probability distribution $n(B)$ of the spatially varying magnetic field is inferred from the muon precession signal [7]. This distribution reflects the arrangement of vortex lines. We measure $n(B)$ as a function of external magnetic field and temperature, calculating the second moment $\left\langle[\Delta B]^{2}\right\rangle$, the third moment $\left\langle[\Delta B]^{3}\right\rangle$, and the related dimensionless line shape anisotropy ratio $\alpha=\left\langle[\Delta B]^{3}\right\rangle^{1 / 3} /\left\langle[\Delta B]^{2}\right\rangle^{1 / 2}$, with the $k$ th moment defined by $\left\langle[\Delta B]^{k}\right\rangle=\sum n\left(B_{i}\right)\left(B_{i}-\langle B\rangle\right)^{k} / \sum n\left(B_{i}\right)$. Figures 1(a) and 1(b) show field distributions for two values of the applied magnetic field. As discussed in Ref. [6], the data 

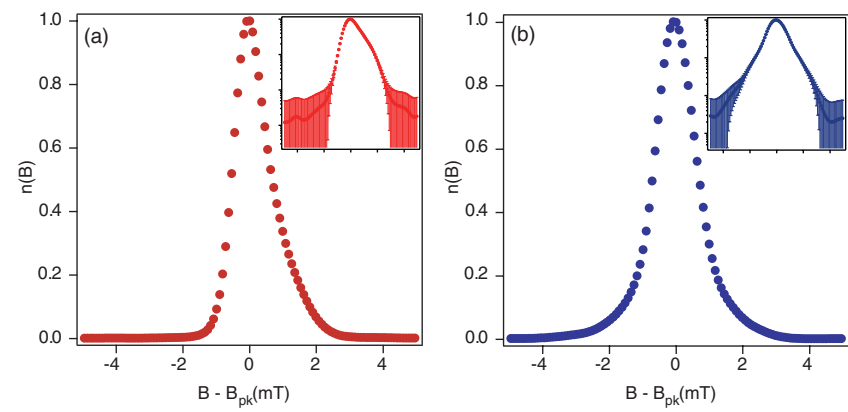

FIG. 1 (color online). The field distribution function $n(B)$ for two values of the applied magnetic field $H$ at $T=5 \mathrm{~K}$ : (a) $H=40 \mathrm{mT}$ and (b) $H=0.5 \mathrm{~T}$. The insets show the same data, plotted over the same range, with the $y$ axis plotted on a logarithmic scale, indicating the errors on the points. The curves are normalized at the mode of the distribution, $B_{p k}$.

at $40 \mathrm{mT}$ [Fig. 1(a)] show a tail on the high-field side and hence positive sign of $\alpha$ expected for a lattice (or Bragg glass) structure. However, the $0.5 \mathrm{~T}$ data [Fig. 1(b)] show a broader and more symmetric distribution in which this tail is absent and the line shape at this field is slightly skewed the opposite way, signaling that the flux-line structure is not ordered like a lattice. Instead, it is in a vortex glass state, which dominates the phase diagram in the inset of Fig. 3.

Figure 2 shows the behavior of the line shape anisotropy ratio $\alpha$ as a function of magnetic field at $5 \mathrm{~K}$ after field cooling; all points lie within the vortex glass phase [6] (inset of Fig. 3). The experimental data are the sequence of black circles; these data are modeled theoretically (see below) by the sequence of red crosses and blue connected squares. Note the reduction in $\alpha$ beginning at relatively low field values, the precipitous change of sign at $B \sim 0.35 \mathrm{~T}$, followed by saturation at an approximately constant negative value. Figure 3 shows the variation of $\alpha$ over the $H-T$ plane, further illustrating a change of sign from positive to negative values that occurs deep within the vortex glass phase. We note that a negative third moment of the field distribution is also observed in the vortex liquid regime; this is an outstanding problem for theories of vortex line correlations $[8,9]$.

For a system of flux lines taken to be rigid along the $z$ axis (field direction), the third moment is obtained from

$$
\left\langle[\Delta B]^{3}\right\rangle \propto \iint d \mathbf{q}_{1} d \mathbf{q}_{2} S^{(3)}\left(\mathbf{q}_{1}, \mathbf{q}_{2}\right) b\left(\mathbf{q}_{1}\right) b\left(\mathbf{q}_{2}\right) b\left(-\mathbf{q}_{1}-\mathbf{q}_{2}\right),
$$

where $S^{(3)}\left(\mathbf{q}_{1}, \mathbf{q}_{2}\right)=\frac{1}{N}\left\langle\delta \rho\left(\mathbf{q}_{1}\right) \delta \rho\left(\mathbf{q}_{2}\right) \delta \rho\left(-\mathbf{q}_{1}-\mathbf{q}_{2}\right)\right\rangle[10]$ is the triplet structure factor and the proportionality constant is $B /(2 \pi)^{4} \Phi_{0}$. Here, $b(\mathbf{q})$ is the field of a single vortex in Fourier space, while $\delta \rho(\mathbf{q})$ is the Fourier component at wave vector $\mathbf{q}$ of the deviation of the flux-line density from its average value. The second moment is related similarly to the two-particle correlation function, i.e., to the conventional structure factor $S(q)=\frac{1}{N} \times$

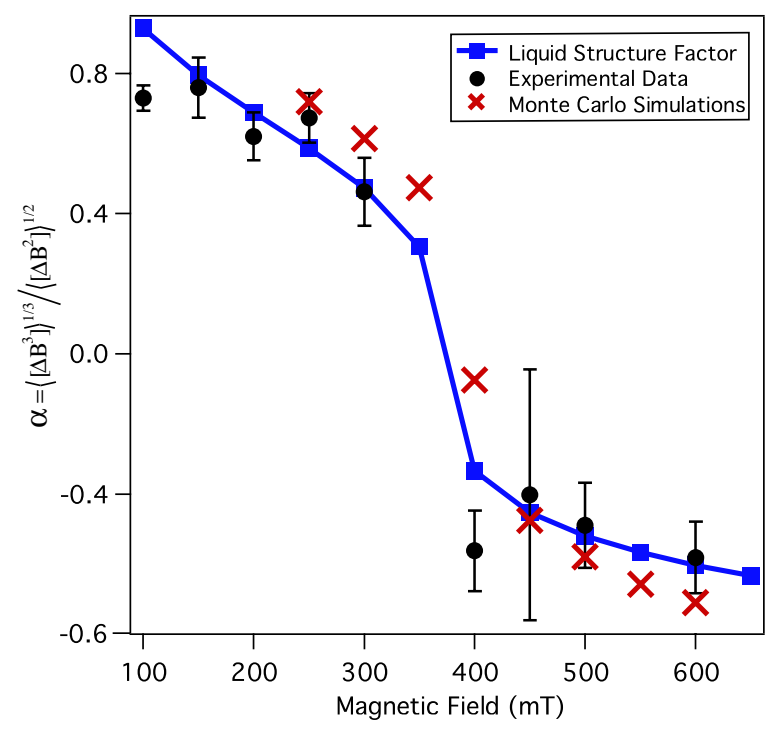

FIG. 2 (color online). The line shape anisotropy ratio $\alpha$ as obtained in (i) experiments at $5 \mathrm{~K}$ (black circles), (ii) calculated from Monte Carlo simulations of disordered structures (red crosses), and from (iii) a theoretical description in terms of liquid state theory (blue squares connected by a solid line).

$\langle\delta \rho(\mathbf{q}) \delta \rho(-\mathbf{q})\rangle$. In these expressions, $N$ is the total number of vortices and the brackets $\langle\cdots\rangle$ denote an average over the sample [11,12]. Note that $S(q) \geq 0$.

The simple London model gives $b(\mathbf{q})=B /\left(1+\lambda^{2} q_{\perp}^{2}\right)$, where $\lambda$ is the $a b$-plane penetration depth and $\mathbf{q}=$ $\left(\mathbf{q}_{\perp}, q_{z}=0\right)$. However, this yields a magnetic induction which diverges at the vortex core. This unphysical divergence is eliminated by multiplying by a "form factor" $f(q)$ [13]. All the available analytic expressions for $f(q)$ give

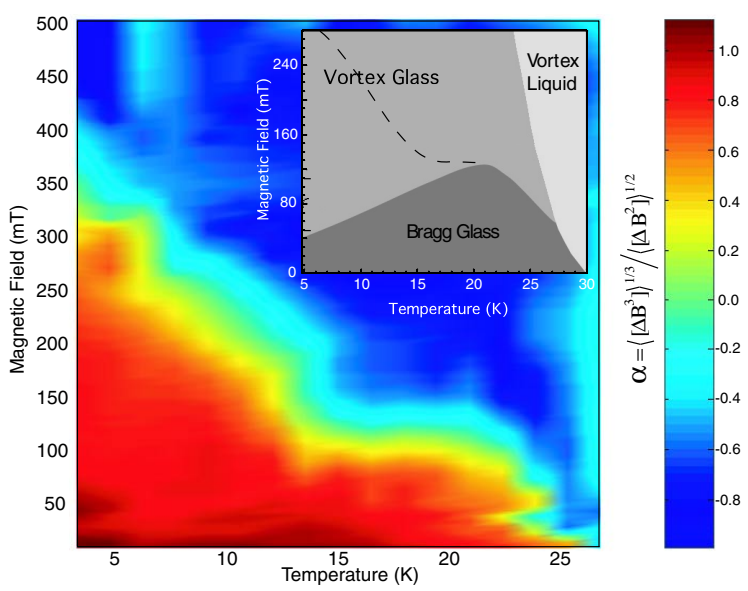

FIG. 3 (color online). Regimes in $H-T$ space associated with a fixed sign of $\alpha=\left\langle[\Delta B]^{3}\right\rangle^{1 / 3} /\left\langle[\Delta B]^{2}\right\rangle^{1 / 2}$, and thus of the third moment. The inset shows the schematic phase diagram for this system in $H-T$ space, obtained from a combination of muon-spin rotation and magnetic and small angle neutron scattering measurements [6]. The change of sign of $\alpha$ reported here occurs deep within the vortex glass phase shown in the inset (dotted line in inset) and over a fairly short range in field and temperature. 
positive values for all $q$ [14]. For a perfect triangular lattice, Eq. (1) reduces to a sum over two sets of reciprocal lattice vectors. Each term in such a sum is manifestly positive. The form factor reduces the value of the third moment, but it cannot change its sign.

We now relax the requirement of a lattice structure. One possibility is to assume uncorrelated lines, i.e., $S(q)=1$, $S^{(3)}\left(\mathbf{q}_{1}, \mathbf{q}_{2}\right)=1$ [11]. This limit yields anomalously large field $\left(B_{0}\right)$ dependent values for the second moment $\left(\left\langle[\Delta B]^{2}\right\rangle=B_{0} \Phi_{0} / 4 \pi \lambda^{2}\right.$, in comparison to the perfect lattice, for which $\left.\left\langle[\Delta B]^{2}\right\rangle=3.71 \times 10^{-3} \Phi_{0}^{2} / \lambda^{4}\right)$. It also leads to a positive third moment. Alternatively, if one applies the "convolution approximation" from the fluid literature [10], one can express $S^{(3)}$ in terms of the twoparticle correlator only: $S^{(3)}\left(\mathbf{q}_{1}, \mathbf{q}_{\mathbf{2}}\right)=S\left(q_{1}\right) S\left(q_{2}\right) S\left(\mid \mathbf{q}_{\mathbf{1}}-\right.$ $\left.\mathbf{q}_{2} \mid\right)$. However, this approach also gives positive $\left\langle[\Delta B]^{3}\right\rangle$ because the integrand in Eq. (1) is manifestly positive. The effects of line wandering can be incorporated using expressions for $S\left(q_{\perp}, q_{z}\right)$ obtained via the boson approximation [2] - numerical values of the moments are reduced but again the sign of $\left\langle[\Delta B]^{3}\right\rangle$ should remain positive [12].

Negative third moments in vortex glass phases which lack long-range order can thus only result from vortex line arrangements with nontrivial three-particle correlations beyond the convolution approximation. To validate these ideas, we have generated simulations of disordered states with these attributes. Simulated annealing techniques were used to find the ground state of a system of 6400 particles (rigid vortex lines) interacting through a potential of the form $\gamma K_{0}(r / \lambda)$, and with a quenched one-body potential arising from 3700 randomly placed pinning sites. The pinning sites were modeled as attractive Gaussian wells of the form $V_{d}(r)=-\gamma^{\prime} e^{-r^{2} / R_{p}^{2}}$, where $R_{p}$ is a length scale for disorder and $\gamma^{\prime}$ gives the strength of the disorder. We took $R_{p}=0.1$ in units of the interparticle separation $a$, and varied $\gamma / \gamma^{\prime}$ in the range [0.001:1000]. Without disorder, the ground state is a crystal. As the strength of pinning is increased, this crystal fragments into smaller domains [15] in a polycrystalline arrangement in which the typical domain size is controlled by the pinning strength [16]. We can then calculate $n(B)$ and its moments by constructing a histogram of local field values [17]. In Fig. 4, we compare field contours obtained for a perfect triangular lattice (a) and a disordered arrangement with the attributes discussed above (b). The disordered case shows the absence of longrange order in both vortex position and field value while maintaining a marked tendency towards local triangular coordination. Explicit calculations of $\alpha$ from a distribution of vortex lines with such a structure are a very good representation of the experimental data in Fig. 2.

The same general result may be obtained by analytic calculations in certain limits. The triplet structure factor is conventionally decomposed as

$$
\begin{aligned}
S^{3}\left(\mathbf{q}_{1}, \mathbf{q}_{2}\right)= & S\left(q_{1}\right) S\left(q_{2}\right) S\left(\left|-\mathbf{q}_{1}-\mathbf{q}_{2}\right|\right) \\
& \times\left[1+\rho^{2} C^{(3)}\left(\mathbf{q}_{1}, \mathbf{q}_{2}\right)\right],
\end{aligned}
$$
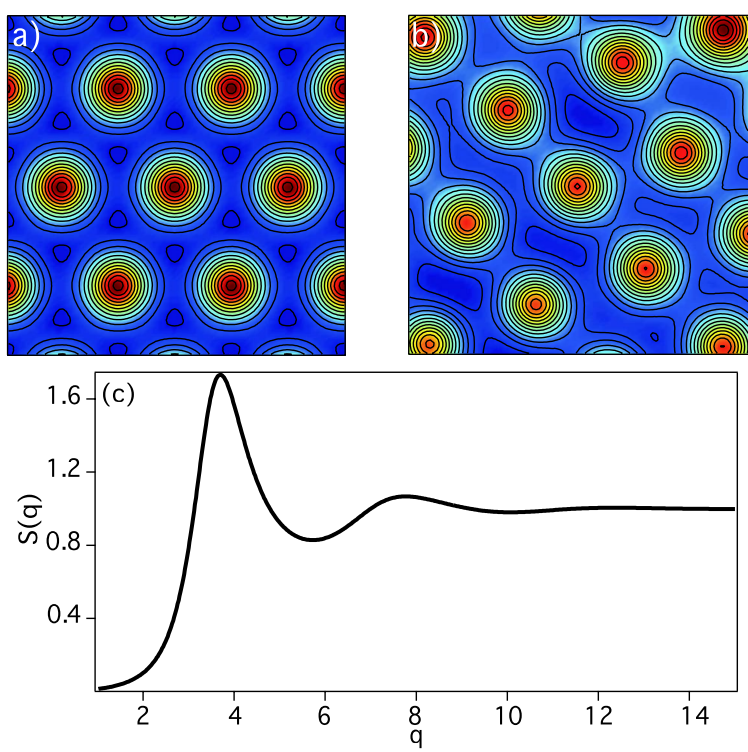

FIG. 4 (color online). Contours of constant magnetic field strength $B(r)$ computed for (a) a perfect Abrikosov lattice at $B=$ $0.5 \mathrm{~T}$ and (b) a disordered state with a translational correlation length of about 4 intervortex spacings generated using simulated annealing techniques, with $\gamma / \gamma^{\prime}=3$ (see text). In (c) is shown a plot of the structure factor $S(q)$, obtained analytically as described in the text ( $q$ is given in units of the inverse mean interparticle spacing).

where $C^{(3)}$ is the triplet direct correlation function [10] and $\rho$ is the average density of the liquid [12]. In our earlier decomposition of $S^{(3)}\left(\mathbf{q}_{1}, \mathbf{q}_{2}\right)$, using the convolution approximation, we assumed $C^{(3)}=0$, an approximation which is clearly inadequate here [18]. To progress beyond the convolution approximation, we assume that the lines are rigid over the scale of the penetration depth and we model translational correlations deep in the vortex glass phase in terms of correlations in an equilibrium fluid [12], using an accurate analytic approximation for $C^{(3)}\left(\mathbf{q}_{1}, \mathbf{q}_{2}\right)$ [19]. We use the pair correlations of the $\gamma K_{0}(r / \lambda)$ potential appropriate to rigid flux lines to describe local structure in the glass phase. These are obtained from solutions to the self-consistent equations of classical liquid state theory in the hypernetted chain approximation [10]. Our model $S(q)$ [Fig. 4(c)] is derived from liquid state computations at a single density $(\lambda / a \sim 4.0$, with $a$ the mean interparticle spacing, corresponding to a field of $0.5 \mathrm{~T}$ and $\lambda \sim 2800 \AA$ ) using a value for the coupling constant $\Gamma=\gamma / k_{B} T$ of 50 . We assume that the local structure of the glass as captured in $S(q)$ is not altered substantially as the field is varied, once all length scales are expressed in units of the mean interparticle spacing $a$, an assumption which should be valid in the limit where $\lambda \gg a$. Using Eqs. (1) and (2), we calculate $\alpha$, illustrated in Fig. 2 as the sequence of connected blue squares. Note that as the field value is increased, the third moment changes sign, with $\alpha$ saturating at a value of about -0.6 , close to the value in the experimental data. This relatively simple analytical model 
thus enables a robust description of the effects of threebody correlations in the vortex glass phase.

Similar behavior is also seen in the highly anisotropic superconductor $\mathrm{Bi}_{2} \mathrm{Sr}_{2} \mathrm{CaCu}_{2} \mathrm{O}_{8+\delta}$ over a range of doping $[8,9]$, suggesting that system-specific interpretations of the negative third moment are unlikely. In Refs. [20,21] a transition from a triangular to a square vortex lattice was observed in the more highly doped system. We find no evidence for this at the field values we probe. At high fields our $\mu$ SR data do not show the tail in $n(B)$ on the high-field side which would signal a crystalline arrangement, whether square or triangular. Magnetic order can coexist with superconductivity in LSCO over a restricted doping range. We have characterized the sample with neutrons and with longitudinal $\mu \mathrm{SR}$, finding magnetic signatures only below

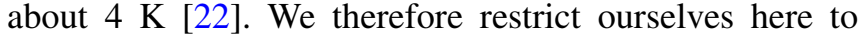
temperatures above this value where any magnetic fluctuations, should they exist, lie well outside the muon time window and cannot contribute to the depolarization.

The sign of the third moment reflects the competition between the (positive) contributions from the vortex cores, which yield the positive tail of $n(B)$, and (negative) contributions from field values at the centers of the triangles formed locally by the vortices and associated with the minima of $n(B)$. Structures with strong local triplet correlations but no long-range order protect both these contributions but subtly enhance the negative ones, since now the positions of particles can fluctuate relative to each other, unlike in the perfect crystal, while retaining a strong tendency to local triangular coordination [see Fig. 4(b)] as in the crystal. The sign changes arises when the negative contributions to the integral [Eq. (1)] overwhelm the positive contributions. The integrand of Eq. (1) varies strongly as a function of $q$ in the vicinity of the first peak of $S(q)$ and below. For larger $q$, form factor cutoffs set in, while at smaller $q$, the integrand becomes negative, due to the generally large and negative value of $C^{(3)}$ in this region (see Ref. [19]). The resultant sign depends on the location of the first peak of $S(q)$, which is itself determined by the magnetic field. Thus, nontrivial three-body correlations arise out of $C^{(3)}$ in a disordered system (which, unlike a crystal, has contributions to the integral at smaller $q$ than the first Bragg peak, i.e., at length scales which are a little larger than nearest neighbor vortex spacings). Although the change in sign of the third moment at high fields confirms the existence of nontrivial three-body correlations, it does not indicate the formation of a new vortex state. Instead, this observation supports our simple theoretical and computational models of structure and correlations in the vortex glass state, from which such a change in sign follows naturally as the magnetic field is varied, without the requirement of a phase transition.

In conclusion, this Letter describes an unusual experimental consequence of many-particle correlations in a magnetic flux-line system, showing how three-body corre- lations are responsible for negative third moments in the field distributions associated with glassy phases of vortex lines. Our results motivate and validate the use of simple analytic approximations to describe three-body correlations in bulk disordered systems, an approach which should find wider application in areas outside the field of superconductivity.

This work was supported by the DST (India), the Swiss National Foundation and NCCR MaNEP, the EPSRC (U.K.), and the Ministry of Education, Science and Technology of Japan. The $\mu$ SR experiments were performed at the Swiss Muon Source, Paul Scherrer Institute, Villigen.

*Present address: Département de Physique, Université de Fribourg, Chemin Du Musée 3, Fribourg CH1700, Switzerland.

${ }^{\dagger}$ Present address: Meteorological Service, Ministry of Agriculture, 28 Nikis Avenue, 1086 Lefkosia, Cyprus.

[1] K. Zahn et al., Phys. Rev. Lett. 91, 115502 (2003).

[2] G. Blatter et al., Rev. Mod. Phys. 66, 1125 (1994).

[3] T. Giamarchi and S. Bhattacharya, in High Magnetic Fields: Applications in Condensed Matter Physics and Spectroscopy, edited by C. Berthier et al. (SpringerVerlag, Berlin, 2002), p. 314.

[4] T. Giamarchi and P. Le Doussal, Phys. Rev. Lett. 72, 1530 (1994).

[5] T. Klein et al., Nature (London) 413, 404 (2001).

[6] U. Divakar et al., Phys. Rev. Lett. 92, 237004 (2004).

[7] J. E. Sonier, J. H. Brewer, and R. F. Kiefl, Rev. Mod. Phys. 72, 769 (2000).

[8] D. R. Harshman et al., Phys. Rev. Lett. 67, 3152 (1991).

[9] T. Blasius et al., Phys. Rev. Lett. 82, 4926 (1999).

[10] J.P. Hansen and I.R. Macdonald, Theory of Simple Liquids (Academic, London, 1986), 2nd ed.

[11] E. H. Brandt, Phys. Rev. Lett. 66, 3213 (1991)

[12] G. I. Menon et al., Phys. Rev. B 60, 7607 (1999).

[13] A. Yaouanc et al., Phys. Rev. B 55, 11107 (1997).

[14] Analytic expressions for $f(q)$ include $f(q)=g K_{1}(g)$, where $g=\sqrt{2} \xi\left(q^{2}+\lambda^{-2}\right)^{1 / 2}$ and $K_{1}(x)$ is a modified Bessel function, the Gaussian form $f(q)=$ $\exp \left(-\xi^{2} q^{2} / 2\right)$, and the exponential $f(q)=\exp (-\sqrt{2} \xi q)$ [13].

[15] G. I. Menon, Phys. Rev. B 65, 104527 (2002).

[16] M. Chandran et al., Phys. Rev. B 69, 024526 (2004).

[17] We evaluate the local field at $10^{6}$ points chosen at random within the simulation box, calculating $n(B)$ and its moments from these measurements.

[18] H. W. Jackson and E. Feenberg, Rev. Mod. Phys. 34, 686 (1962).

[19] S. Zhou and E. Ruckenstein, Phys. Rev. E 61, 2704 (2000).

[20] R. Gilardi et al., Phys. Rev. Lett. 88, 217003 (2002).

[21] A. J. Drew et al., Physica (Amsterdam) 374B, 203 (2006).

[22] See EPAPS Document No. E-PRLTAO-97-015644 for a supplemental figure. For more information on EPAPS, see http://www.aip.org/pubservs/epaps.html. 